\title{
La viveza de imagen y los juicios metamnemónicos en las personas mayores
}

\author{
Imagery vividness and metamnemonic judgments in the elderly
}

\author{
Leticia Ameijide, Alfredo Campos \\ Departamento de Psicología Social, Básica y Metodología, Universidad de Santiago de Compostela, España
}

\begin{abstract}
Resumen
El objetivo de este estudio era valorar la viveza de imagen de las personas mayores en el recuerdo, los juicios metamnemónicos (juicios de facilidad de aprendizaje, juicios de aprendizaje y juicios de confianza) y las puntuaciones gamma. La muestra estaba compuesta por un grupo de 50 mujeres entre 65 y 86 años con una media de edad de 75.02 años $(S D=5.86)$. Ellas aprendieron la traducción de una lista corta de 16 palabras latinas mediante la mnemotecnia de la palabra clave reforzada con dibujos y también, cubrieron el Betts'QMI. Los resultados indicaron que el recuerdo mostró una diferencia marginalmente significativa y la viveza de imagen influyó en las puntuaciones gamma entre los juicios de facilidad de aprendizaje y el recuerdo.

Palabras clave: viveza, juicios metamnemónicos, mayores, imágenes mentales, palabra clave.
\end{abstract}

\begin{abstract}
The aim of this study was to assess the elderly's imagery vividness in the recall, metamnemonic judgments (ease of learning judgments, judgments of learning and confidence level) and gamma scores. The sample was composed by a group of 50 women between 65 and 86 years old with a mean age of 75.02 years $(S D=5.86)$. They learned the translation of a short list of 16 Latin words by the keyword mnemonics reinforced with drawings and also, they filled in the Betts'QMI. The results indicated that the recall showed a marginally significant difference and the imagery vividness had an influence in gamma scores between ease of learning judgments and the recall. Keywords: vividness, metamnemonic judgments, elderly, mental imagery, keyword.
\end{abstract}

La mnemotecnia se define como el conjunto de métodos que ayudan a la memoria (Campos, 1998) y se divide en reglas y sistemas mnemotécnicos (Higbee, 1998). Las reglas tienen un único objetivo y se usan para recordar datos específicos; mientras que los sistemas se centran en objetivos generales para recordar diferentes conjuntos de elementos (Higbee, 1998).

En 1975, Atkinson desarrolló la mnemotecnia de la palabra clave o "keyword", la regla mnemotécnica más utilizada para el aprendizaje de vocabulario de un segundo idioma. Este método se basa en dos pasos. En el primero, "verbal", se elabora una palabra clave concreta y con semejanza fonética u ortográfica a la palabra extranjera que se debe aprender. En el segundo paso, "visual", se crea una imagen mental relacionando la palabra clave con el significado de la palabra extranjera (Campos, Amor y González, 2002; Campos, Camino y
Pérez-Fabello, 2011). La mnemotecnia de la palabra clave resultó eficaz en los estudios de laboratorio cuando el experimentador presenta la palabra clave y la aplicación es individual (González, Amor y Campos, 2003). En los últimos años, Campos y colaboradores (Campos et al., 2002; Campos, Amor y González, 2004a, 2004b) idearon una nueva propuesta en sus estudios que consistió en que los compañeros de los participantes experimentales (similares en nivel educativo, edad y desarrollo cognitivo) creen la palabra clave. Este método tiene la ventaja de que la palabra clave evocada será la que le salga más espontáneamente a los participantes con el fin de mejorar el recuerdo de la traducción española. En definitiva, esta estrategia de aprendizaje fue superior a otros métodos como el de repetición o el habitual en personas adultas y mayores (Campos y Ameijide, 2014; Campos, Camino y Pérez-Fabello, 2010; Campos et al., 2011; Campos, Pérez-Fabello y Camino, 2010).

Existen diferentes aspectos que influyen en el recuerdo del material de aprendizaje. Uno de ellos es la capacidad de las personas para crear imágenes mentales. Alesandrini (1982) plantea que cuanto mayor es la viveza de imagen, mayor efecto tendrá sobre la memoria debido a que cuando se crea una imagen mental se utiliza el código visual de forma independiente al verbal. En consecuencia, al usar los dos códigos en conjunto según la Teoría de la Codificación Dual de Paivio (1975), existen más posibilidades de recuerdo de la información. El otro aspecto es la metamemoria (Ameijide y Campos, 2011a, 2011b; Campos y Ameijide, 2011, 2014) que se basa en el conocimiento y el control que posee el individuo sobre el funcionamiento de su memoria, incluyendo sus distintas fases: codificación, almacenamiento y recuperación (Ruiz, 2008).

Dentro del modelo general de memoria, Nelson y Narens (1990) plantean el estudio de la metamemoria en tres etapas principales: adquisición (antes del aprendizaje propiamente dicho), retención (mantenimiento del conocimiento previamente adquirido) y recuperación consciente de la información. Los juicios metamnemónicos se pueden llevar a cabo en cuatro momentos del proceso de aprendizaje: a) antes del proceso de adquisición (juicios de facilidad de aprendizaje, EOL = ease of learning judgments), b) durante o inmediatamente después de la adquisición, pero antes de la prueba de recuperación (juicios de aprendizaje, JOL = judgments of learning) y c) después de la recuperación del material durante la prueba (juicios de confianza, $\mathrm{CL}=$ confidence level judgments).

Para evaluar la precisión de los juicios metamnemónicos se calcula una medida de exactitud 
relativa mediante la correlación gamma de Kruskal-Goodman entre las puntuaciones de cada participante en cada uno de los juicios y su recuerdo (Nelson y Dunlosky, 1991, 1992; Overschelde y Nelson, 2006).

El objetivo de este estudio era valorar la influencia de la viveza de imagen de las personas mayores en el recuerdo, los juicios metamnemónicos (juicios de facilidad de aprendizaje, juicios de aprendizaje y juicios de confianza) y las puntuaciones gamma.

\section{Método}

\section{Participantes}

La muestra estaba compuesta por un grupo de 50 mujeres entre 65 y 86 años con una media de edad de 75.02 años $(S D=5.86)$. Las participantes pertenecían a diferentes Centros de Mayores de Galicia, tenían un nivel de estudios básicos, no padecían deterioro cognitivo y se presentaron voluntarias para esta investigación.

\section{Instrumentos}

Se utilizó una lista corta de 16 palabras latinas ( 8 altas en viveza de imagen y 8 bajas en viveza de imagen), con sus respectivas palabras clave, las traducciones españolas de las palabras latinas, las frases interactivas entre cada palabra clave y el significado de la palabra latina y finalmente, 16 dibujos que representaban el significado de dichas frases interactivas. Como entrenamiento se emplearon 4 palabras latinas ( 2 altas en viveza de imagen y 2 bajas en viveza de imagen).

Por último, se usó la versión española (Campos y Pérez-Fabello, 2005) de la forma abreviada del Betts' Questionnaire Upon Mental Imagery (Betts'QMI) (Sheehan, 1967). Es un test destinado a cuantificar la viveza de imagen que experimenta un individuo y contiene cinco ítems de cada una de las siete modalidades sensoriales. Los participantes debían valorar cada ítem en una escala que oscila entre 1 y 7 puntos. Altas puntuaciones en el test indican baja viveza de imagen y viceversa.

\section{Procedimiento}

En primer lugar, se seleccionaron un conjunto de 24 palabras latinas, 12 altas en viveza de imagen y 12 bajas en viveza de imagen, de las publicadas por Valle (1998). A continuación, se consideraron palabras altas en viveza de imagen las que tenían una puntuación superior a 5.20 y palabras bajas en viveza de imagen las que tenían una puntuación inferior a 4.21. Estas puntuaciones se obtuvieron al sumar y restar a la media, 2/5 de la desviación típica, según el estudio de Valle (1998). Posteriormente, se tradujeron las palabras al latín y se rechazaron aquellos vocablos que sonasen o se escribiesen iguales o muy parecidos en los dos idiomas (latín y español). Se escogió el latín porque era un idioma desconocido para las participantes y así, partían del mismo nivel de conocimientos.
A continuación, esta lista de 24 palabras latinas se le presentó a un grupo de 15 participantes elegidos al azar, de la misma edad y características socioculturales que los participantes experimentales para que, sin límite de tiempo, elaborasen las palabras clave (palabras españolas), lo más concretas posible y que sonasen o se escribiesen lo más parecido posible a las palabras latinas. Después, debían crear una frase para cada palabra en la que aparecía reflejada la relación de interacción entre la palabra clave y la traducción española de la palabra latina. Posteriormente, los investigadores seleccionaron las palabras clave de mayor frecuencia de aparición y las frases interactivas más repetidas. Por ejemplo, palabra latina "Permixtio", palabra clave "Permiso", traducción española "Mezcla" y frase interactiva "Pedí permiso para realizar la mezcla”. Finalmente, se rechazaron las palabras clave menos frecuentes hasta quedar con una lista definitiva de 16 palabras latinas ( 8 altas en viveza de imagen y 8 bajas en viveza de imagen) que fueron las que se presentaron a las participantes como objetivo de aprendizaje en el transcurso del experimento. La media de imagen de las palabras altas en imagen fue de 6.27 $(S D=.35)$ y la media de las palabras bajas en imagen fue de $3.55(S D=.54)$. La media en viveza de imagen del total de las palabras fue de $4.91(S D=1.47)$. La diferencia en viveza de imagen entre las palabras altas en imagen y las bajas en imagen fue significativa, $t(1,14)=-11.91, p<.001)$. También se seleccionaron 4 palabras latinas ( 2 altas en viveza de imagen y 2 bajas en viveza de imagen) para el entrenamiento.

A 50 participantes entre 65 y 86 años se les presentó individualmente, en un ordenador conectado a un cañón, 16 diapositivas en las que aparecían la palabra latina, la palabra clave, la traducción española de la palabra latina, la frase interactiva y un dibujo reflejando esa interacción para que la participante las aprendiese mediante la mnemotecnia de la palabra clave reforzada con dibujos. La experimentadora leía dos veces, en voz alta, las tres palabras y la descripción de una imagen interactiva entre la palabra clave y el significado de la palabra latina. El ritmo de presentación de cada diapositiva fue de 15 segundos.

Una vez que se le presentaron a la participante las 4 palabras de entrenamiento, las escribía en un folio, en el cual aparecía la palabra latina y a su lado, tenía que escribir la traducción española. En otro folio, grapado al anterior, se presentaba la lista corta de palabras, que posteriormente se mostraría, al azar, en la pantalla del ordenador para que cada participante escribiera al lado de cada par (palabra latina y traducción española), el porcentaje de facilidad de aprender, (juicios de facilidad de aprendizaje $=\mathrm{EOL}=$ ease of learning), una vez que se presente cada palabra dos veces, siguiendo el modo de aprendizaje visto en el entrenamiento. Cada par se puntúa en una escala de 0 a 100 , siendo las únicas puntuaciones posibles: 0, 20, 40, 60, 80, 100. El 0 indica "muy difícil de aprender" y el 100 "muy fácil de aprender". Dado que la participante era muy lenta a la hora de contestar, se le indicó que diera la respuesta que primero le viniera a la mente, lo más rápido posible. 
Una vez cubierto el juicio de facilidad de aprendizaje, se retira el folio y se pasa la lista de las 16 palabras, siguiendo el mismo procedimiento que en el entrenamiento. Una vez presentados los pares de palabras para el aprendizaje y antes de mostrar la lista para el recuerdo, se presentaron, al azar, las palabras estímulo (palabras latinas) para que cada participante escribiera al lado de cada palabra, el porcentaje de probabilidad de recordar el significado de la palabra latina (juicios de aprendizaje $=\mathrm{JOL}=$ judgments of learning). Cada uno de los ítems o palabras latinas se puntúa en una escala de 0 a 100 , siendo las únicas puntuaciones posibles: $0,20,40,60,80,100$. El 0 indica "estoy completamente seguro de que no la recordaré" y el 100 indica "estoy completamente seguro de que la recordaré". Al igual que en el caso anterior, debido a la lentitud en contestar a lo que se le pedía, se orientó, a cada participante, para que diera la respuesta que primero le viniera a la mente, lo más rápido posible.

Finalizado el juicio de aprendizaje, se retira el folio y se entrega otro con las palabras latinas para que escribiera al lado de cada palabra la traducción española. Al mismo tiempo, tenía que escribir al lado de cada respuesta que dio, el nivel de confianza que poseía en que sus respuestas fuesen las correctas (juicios de confianza $=\mathrm{CL}=$ confidence level). La participante, según va poniendo la respuesta al lado de cada palabra latina, puntúa en una escala de 1 a 5, el nivel de confianza o de seguridad de que dicha respuesta escrita fuese la correcta. El 1 indica "estoy completamente seguro de que la respuesta es incorrecta" y el 5 "estoy completamente seguro de que la respuesta es correcta". Dado que la participante era muy lenta a la hora de contestar, se le indicó que diera la respuesta que primero le viniera a la mente, lo más rápido posible. Una vez cubiertas las hojas de respuesta del recuerdo y del juicio de confianza (CL) finalizaba el experimento.

Para concluir, otro día se presentó a cada participante la versión española (Campos y Pérez-Fabello, 2005) de la forma abreviada del Betts' Questionnaire Upon Mental Imagery (Betts'QMI) (Sheehan, 1967).

\section{Resultados}

Para comprobar si la viveza de imagen influía en el recuerdo de la traducción de las palabras latinas, se efectuó una prueba $t$ de Student para muestras independientes y se encontró que las participantes con baja puntuación en el Betts'QMI (alta viveza de imagen) $(M=11.07, S D=2.35)$ tuvieron un mayor recuerdo que las personas con alta puntuación en el Betts'QMI (baja viveza de imagen) $(M=9.80, S D=2.24)$, mostrando así una diferencia marginalmente significativa, $t(48)=1.90$, $p=.06$.

También se observó si la capacidad para crear una imagen mental vívida influía en todos los juicios metamnemónicos. Los resultados demostraron que no existían diferencias significativas entre las personas con baja puntuación en el Betts'QMI (alta viveza de imagen) y las participantes con alta puntuación en el Betts'QMI (baja viveza de imagen) en ninguno de los juicios metamnemónicos (juicios de facilidad de aprendizaje, juicios de aprendizaje y juicios de confianza).

Finalmente, se trató de averiguar si la viveza de imagen influía en las puntuaciones gamma obtenidas entre cada uno de los juicios metamnemónicos y el recuerdo. Para ello, se realizó una prueba $t$ de Student para muestras independientes y únicamente se halló que las participantes con baja puntuación en el Betts'QMI (alta viveza de imagen) $(M=.46, S D=.42)$ tuvieron una mayor correlación entre los juicios de facilidad de aprendizaje y el recuerdo que las personas con alta puntuación en el Betts'QMI (baja viveza de imagen) $(M=.15, S D=.49)$, reflejando una diferencia significativa, $t(46)=2.35, p=.02$.

\section{Discusión de resultados}

En esta investigación se analizó la viveza de imagen de las personas mayores en el recuerdo, los juicios metamnemónicos (juicios de facilidad de aprendizaje, juicios de aprendizaje y juicios de confianza) y las puntuaciones gamma.

Los resultados indicaron que las participantes con baja puntuación en el Betts'QMI (alta viveza de imagen) obtuvieron un mayor recuerdo que las personas con alta puntuación en el Betts'QMI (baja viveza de imagen). Esto confirma los estudios de Alesandrini (1982), Ameijide y Campos (2011a, 2011b) y de Campos et al. $(2010,2011)$ que también demostraron que cuanto mayor es la viveza de imagen de los mayores, existen más posibilidades de recuerdo de la información.

Además, se observó que la capacidad para crear una imagen mental vívida no influía en ninguno de los juicios metamnemónicos. Este hallazgo no coincide con el estudio obtenido por Ameijide y Campos (2013a) en el que se comprobó que los mayores con alta viveza de imagen obtenían mayores juicios de facilidad de aprendizaje (EOL). Tampoco estuvieron de acuerdo Ameijide y Campos (2013b) ya que ellos demostraron que cuanto mayor es la viveza de imagen, mayores son los juicios de aprendizaje (JOL).

Y finalmente, las participantes con baja puntuación en el Betts'QMI (alta viveza de imagen) consiguieron una mayor correlación entre los juicios de facilidad de aprendizaje y el recuerdo que las personas con alta puntuación en el Betts'QMI (baja viveza de imagen). Este resultado está en la línea de las investigaciones realizadas por Ameijide y Campos (2011a, 2011b) donde se comprobó una correlación mayor entre los juicios de facilidad de aprendizaje y el recuerdo cuando los mayores aprendieron palabras altas en viveza de imagen. La única diferencia que existió entre este estudio y los dos anteriores, fue que las mujeres consiguieron un mejor resultado que los hombres. 


\section{Referencias}

Alesandrini, K. L. (1982). Imagery eliciting strategies and meaningful learning. Journal of Mental Imagery, 6, 125-140.

Ameijide, L., y Campos, A. (2011a). Diferencias individuales en la metamemoria cuando se utiliza la mnemotecnia de la palabra clave. Actas del XI Congreso Internacional Galego-Portugués de Psicopedagoxía (pp. 4191-4201). A Coruña: Universidad de A Coruña.

Ameijide, L. y Campos, A. (2011b). Juicios metamnemónicos sobre la mnemotecnia de la palabra clave: estudio con personas mayores. Actas del XI Congreso Internacional Galego-Portugués de Psicopedagoxía (pp. 4203-4211). A Coruña: Universidad de A Coruña.

Ameijide, L. y Campos, A. (2013a). Percepción de facilidad de aprender (EOL) listas de palabras mediante la mnemotecnia de la palabra clave con dibujos. En R. González, R. Fernández, F. Fariña, M. Vilariño y C. Freire (Eds.), Psicología y Salud I. Educación, aprendizaje y salud (pp. 103-111). A Coruña: GEU Editorial.

Ameijide, L. y Campos, A. (2013b). Influencia de la viveza de imagen en los juicios de aprendizaje (JOL) utilizando la mnemotecnia de la palabra clave reforzada con dibujos. En R. González, R. Fernández, F. Fariña, M. Vilariño y C. Freire (Eds.), Psicología y Salud I. Educación, aprendizaje y salud (pp. 113-120). A Coruña: GEU Editorial.

Atkinson, R. C. (1975). Mnemotechnics in second-language learning. American Psychologist, 30, 821-828.

Campos, A. (Ed.) (1998). Imágenes mentales. Santiago de Compostela: Servicio de Publicaciones de la Universidad de Santiago de Compostela.

Campos, A., y Ameijide, L. (2011). Exactitud de los juicios de aprendizaje (JOLs) y mnemotecnia de la palabra clave en adultos y ancianos. International Journal of Psychological Research, 4, 64-71.

Campos, A., y Ameijide, L. (2014). Mnemotecnia de la palabra clave con dibujos y juicios metamnemónicos de personas mayores. Revista Iberoamericana de Psicología y Salud, 5, 23-38.

Campos, A., Amor, A., y González, M. A. (2002). Presentation of keywords by means of interactive drawings. The Spanish Journal of Psychology, 5, 102-109.

Campos, A., Amor, A., y González, M. A. (2004a). The importance of the keyword- generation method in keyword mnemonics, Experimental Psychology, 51, 125-131.

Campos, A., Amor, A., y González, M. A. (2004b). Drawing-assisted strategies in keyword mnemonics. Studia Psychologica, 46, 211-218.

Campos, A., Camino, E., y Pérez-Fabello, M. J. (2010). Aprendizaje de vocabulario con alta y baja viveza de imagen mediante la mnemotecnia de la palabra clave.
Revista Galego-Portuguesa de Psicoloxía e Educación, 18, 67-77.

Campos, A., Camino, E., y Pérez-Fabello, M. J. (2011). Using the keyword mnemonics method among adult learners. Educational Gerontology, 37, 327-335.

Campos, A., y Pérez-Fabello, M. J. (2005). The Spanish version of Betts' Questionnaire Upon Mental Imagery. Psychological Reports, 96, 51-56.

Campos, A., Pérez-Fabello, M. J., y Camino, E. (2010). Eficacia de la mnemotecnia de la palabra clave en personas adultas. Psicothema, 22, 752-757.

González, M. A., Amor, A., y Campos, A. (2003). La mnemotecnia de la palabra clave. A Coruña: Servicio de Publicaciones de la Universidad de A Coruña.

Higbee, K. L. (1998). Su memoria: Cómo funciona y cómo mejorarla. Barcelona: Paidós.

Nelson, T. O., y Dunlosky, J. (1991). When people's judgments of learning (JOLs) are extremely accurate at prediction subsequent recall: The "delayed-JOL effect”. Psychological Science, 2, 267-270.

Nelson, T. O., y Dunlosky, J. (1992). How shall we explain the delayed-judgment-of-learning effect? Psychological Science, 2, 267-270.

Nelson, T. O., y Narens, L. (1990). Metamemory: A theoretical framework and new findings. En G. H. Bower (Ed.), The psychology of learning and motivation: Advances in research and theory (pp. 125-173). Nueva York: Academic Press.

Overschelde, J. P. V., y Nelson, T. O. (2006). Delayed judgments of learning cause both a decrease in absolute accuracy (calibration) and an increase in relative accuracy (resolution). Memory and Cognition, 7, 1527-1538

Paivio, A. (1975). Imagery and long-term memory. En A. Kennedy y A. Wilkes (Eds.), Studies in long term memory (pp. 57-88). London: Wiley.

Ruiz, M. (2008). Las caras de la memoria. Madrid: Pearson Educación, S. A.

Sheehan, P. W. (1967). A shortened form of Betts' Questionnaire Upon Mental Imagery. Journal of Clinical Psychology, 23, 386-389.

Valle, F. (1998). Normas de imaginabilidad. Oviedo: Servicio de Publicaciones de la Universidad de Oviedo. 